\title{
Embryonal rhabdomyosarcoma of the uterine cervix: a rare case report
}

\author{
Ariana R. Gomes*, Pedro B. Leite, Juliana S. Rocha, Pedro V. Enes, Joana I. Carvalho, \\ Ana R. Pinto, Fernanda M. Costa
}

Department of Obstetrics and Gynaecology, Centro Hospitalar Tâmega e Sousa, Penafiel, Portugal

Received: 23 October 2016

Accepted: 23 November 2016

*Correspondence:

Dr. Ariana R. Gomes,

E-mail: arianafgomes@gmail.com

Copyright: ( ) the author(s), publisher and licensee Medip Academy. This is an open-access article distributed under the terms of the Creative Commons Attribution Non-Commercial License, which permits unrestricted non-commercial use, distribution, and reproduction in any medium, provided the original work is properly cited.

\begin{abstract}
Embryonal rhabdomyosarcoma of the female genital tract is a rare tumour. It tends to occur during childhood in the vagina and, rarely, it can arise in the uterine cervix, with a peak incidence in the second decade. We report a case of a 18-year-old female with an embryonal rhabdomyosarcoma (sarcoma botryoides) presenting himself as a cervical polyp. This tumour consisted of rhabdomyoblasts with miscellaneous differentiation surrounded by a loose, myxoid stroma. The patient was successfully treated with cervical conization and adjuvant chemotherapy. She is now diseasefree at the 28th month follow-up. Awareness of this uncommon lesion in the cervix and its clinical implications is important to prevent misdiagnosis. Therapy has recently inclined to conservative and fertility-sparing treatment.
\end{abstract}

Keywords: Adolescent neoplasm, Embryonal rhabdomyosarcoma, Sarcoma botryoides, Uterine cervix neoplasm

\section{INTRODUCTION}

Embryonal rhabdomyosarcoma (ERMS) is a highly malignant tumour arising from embryonal mesenchymal cells committed to develop into striated muscles and is the most frequent soft tissue sarcoma in childhood and young adults, accounting for 4-6\% of all malignancies in this age group. ${ }^{1}$ The head and neck were the most frequent sites of origin $(35 \%)$, followed by the genitourinary tract (25\%), extremities, trunk, retroperitoneum, and uncommon regions (intrathoracic, gastro-intestinal tract, perianal and anal regions). One of the least common sites for rhabdomyosarcoma in the genitourinary tract is the uterine cervix, but its occurrence has been well documented in the study of 13 cases. $^{2}$ Vaginal primaries are five times more common than cervical primaries. ${ }^{3}$ The mean age at diagnosis of patients with ERMS arising in the cervix is higher than of patients with vaginal lesions. Although ERMS has been described in females as young as 5 months of age, it tends to appear in an older age group (children or even young adults) than those occurring in the vagina. ${ }^{1,3}$
The Intergroup Rhabdomyosarcoma Study Group (IRSG) has reported a new classification of ERMS distinguishing three major histological subtypes: embryonal, alveolar and undifferentiated. The embryonal subtype is the most common, accounting for $68 \%$ of all ERMS cases, with classic, botryoid and spindle cell variants embracing 49, 6 and $3 \%$, respectively. ${ }^{1,3,4}$ Sarcoma botryoides of the cervix uteri rarely occurs in fertile age since it only accounts for $0.2 \%$ of all malignant tumours of the uterus. ${ }^{4}$ This sarcoma generally presents as soft nodules that fill and sometimes protrude from the vagina, resembling a bunch of grapes. ${ }^{1,3}$ This histological subtype is associated with a very favourable outcome. The alveolar type accounts for $20 \%$ of children with ERMS and has a particularly poor prognosis. Undifferentiated ERMS is the rarest type and is also associated with a poor prognosis. $^{3}$

The prognosis of these lesions was poor prior to the introduction of effective adjuvant chemotherapy. ${ }^{1}$ A combined modality approach to treat ERMS using surgery, chemotherapy and radiotherapy has evolved over 
the past years and has improved survival rates to more than $60 \%$ for all stages of any site, including genitourinary tumours, and up to $90 \%$ for localized disease. $^{3}$

We report a case of sarcoma botryoides of the cervix presenting as a cervical polyp in an 18-year-old female, treated with conservative surgery and adjuvant chemotherapy.

\section{CASE REPORT}

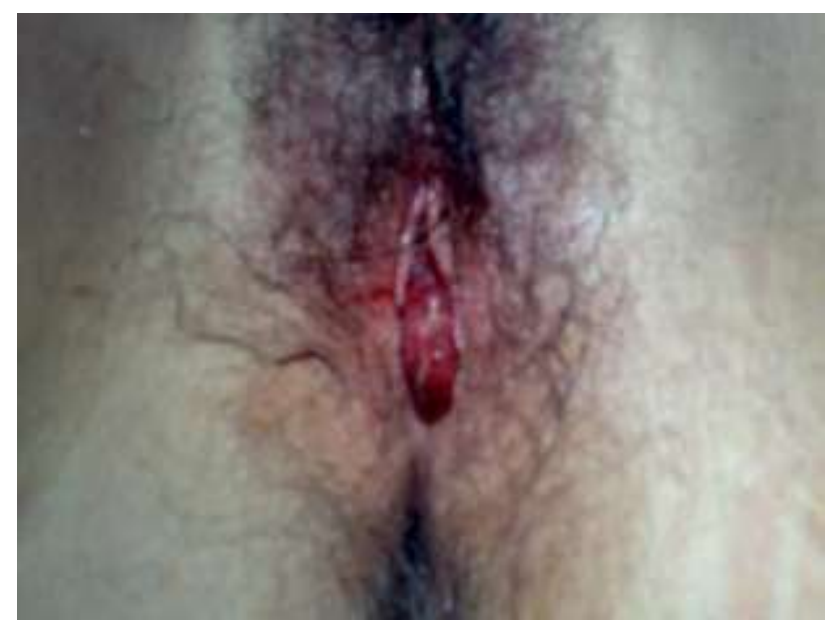

Figure 1: Polypoid tumour of $\mathbf{5} \mathbf{~ c m ~ i n ~ d i a m e t e r ~ w i t h ~ a ~}$ smooth surface filling the vaginal cavity and protruding from the vaginal introitus.

An 18-year-old null gravid female presented with vaginal spotting for 5 months and the sensation of a mass protruding from the introitus. The medical history was negative for relevant pathologies or genetic syndrome. Family history was also irrelevant. The gynaecological and obstetric anamnesis was normal, with menarche at age 12, subsequent cycles regular for rhythm, quantity and length; the patient never had sexual activity. Pelvic examination immediately revealed a grape-like polypoid tumour of $5 \mathrm{~cm}$ in diameter with a smooth surface, arising through a thin stalk from the anterior lip of the cervix, filling the vaginal cavity and protruding from the introitus [Figure 1]. A polypectomy was performed.

The specimen received for pathological examination was a $5 \mathrm{~cm}$ lobulated mass covered by pink, smooth and glistening mucosa. No distinct stalk was identified [Figure 2]. On microscopic examination, the mass was extensively ulcerated and made of spindle mesenchymal cells of average and small size, surrounded by a myxoid stroma [Figure 3]. Immunohistochemical staining, highlighted positive cells for desmin, vimentin and myogenin and negative cells for estrogen and progesterone receptors. A few areas of metaplasic cartilaginous differentiation were also present. These findings were consistent with an ERMS (sarcoma botryoides) of the uterine cervix. After the results of the pathologic examination were communicated to the patient, a computed tomography scan of the chest and a nuclear magnetic resonance of the abdomen and pelvis were performed which revealed no evidence of metastatic or residual disease. The patient was submitted to a cervical conization and no residual tumour was identified in the cervical conization specimen. According to the IRSG criteria, this tumour was staged as group IA. She received adjuvant chemotherapy, consisting of 4 cycles of IVA pattern (Ifosfamide $3 \mathrm{~g} / \mathrm{m}^{2}$ x 2 days - Vincristine $1,5 \mathrm{mg} / \mathrm{m}^{2}$ x 1 day - Actinomycin $1,5 \mathrm{mg} / \mathrm{m}^{2}$ x 1 day) + Mesna and further 5 cycles of Vincristine and Actinomycin. To date, the patient is at the $3^{\text {th }}$ year followup, in complete remission without clinical signs of ovarian failure.

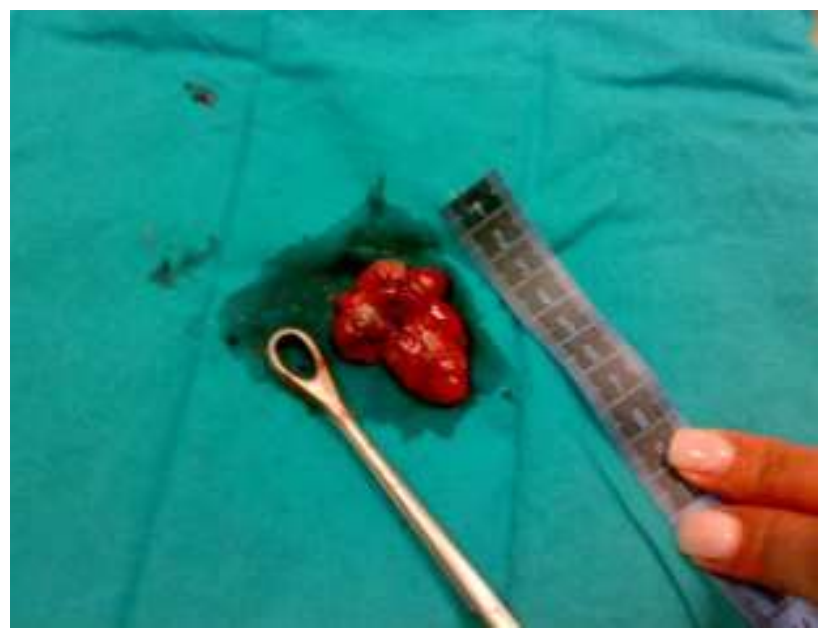

Figure 2: Lobulated mass, measuring 5 centimeters in diameter, covered by pink, smooth and glistening mucosa.

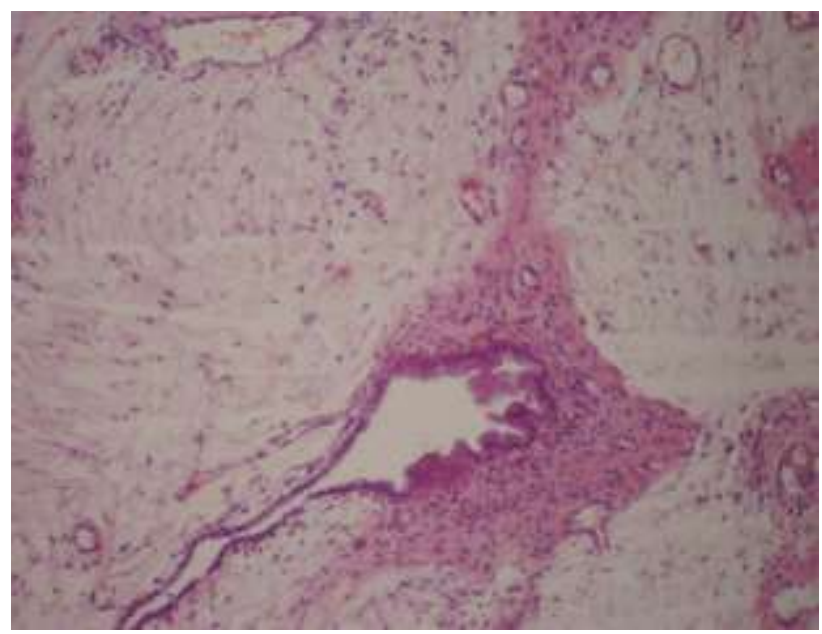

Figure 3: Microscopic examination (hematoxylin and eosin stain): Spindle mesenchymal cells of average and small size, surrounded by a myxoid stroma.

\section{DISCUSSION}

Sarcoma botryoides in the cervix is rare and most of our information is based on individual case reports. Currently 
the largest series consisting of original cases included 14 patients. ${ }^{5}$ Furthermore, information regarding this tumour is difficult to obtain from the literature because the primary place of origin is not always known or described. ${ }^{4}$

Usually, it looks like a polypoid fragile mass, measuring around $2-9.5 \mathrm{~cm}$ of diameter and is always associated with vaginal bleeding and sometimes a feeling of a mass in the vaginal introitus. Like sarcoma botryoides occurring at other mucosal sites, the tumour may form soft, grape-like clusters, present as single or multiple polyps. The microscopic findings consist of rhabdomyoblasts of variable differentiation dispersed within a loose, myxoid stroma. Several cases, including ours, have shown metaplasic cartilaginous differentiation. However, a precise diagnosis is sometimes difficult, because it macroscopically resembles a benign polyp and, in about $40 \%$ of cases, it can histologically appear as a few cell mass with an edematous and laxus stroma, being confusing for pathologists. ${ }^{4}$ Several other lesions enter into the pathologic differential diagnosis, including adenosarcoma, rhabdomyoma, edematous cervical mesodermal polyps (pseudosarcoma botryoides), and other "small, round, blue cell" tumours. ${ }^{6}$ Adenosarcomas, a strong option that was considered in our case as a differential diagnosis, can also be found in young female, but histologically can be distinguished from sarcoma botryoides by their fibrous stroma and leaf-like pattern of glands. Also, immunohistochemical markers that usually label ERMS are desmin, with a positivity rate of 75$100 \%$, vimentin and myogenin, which is considered the most sensible and specific marker (70-100\% of cases). ${ }^{4,6}$

Before 1970, radical surgery was considered the gold standard. However, after consecutive trials, the IRSG has modified the treatment protocols with the addition of multiple chemotherapeutic agents to surgery, greatly improving the prognosis and survival of thistumour and also allowed more conservative fertility-sparing surgeries in early stages of the disease. ${ }^{1,4,5,7}$ Consequently, localized female genital tract disease normally is curable with combination of chemotherapy, a conservative surgical approach and the use of radiotherapy for selected patients. ${ }^{4}$ Nevertheless the extent of surgical therapy and subsequent adjuvant therapy should be balanced between the patient's desire to maintain fertility and the presence of unfavourable prognostic variables, such as extensive uterine involvement and/or metastasis, deep myometrial invasion, lymphatic invasion and foci of alveolar subtype. ${ }^{8}$ The presence of these factors should lead to discussion with the patient and eventually their parents relative to more aggressive treatment. ${ }^{1,4,7}$

In the case reported, after a multidisciplinary team evaluation, we have decided to perform fertility-sparing surgery followed by chemotherapy for the treatment of this tumour.
More recent studies also report successfully treatment of these tumours with fertility-sparing surgery with an innovating way: cervical conization or robotic-assisted radical trachelectomy in combination with multi-agent chemotherapy. 9

Over recent years, the approach of ERMS, like other malignant diseases have been increasingly conservative in the sense of the preservation of fertility, given its prevalence in young nulliparous. ${ }^{5,8,9}$ This case represents another example of successful conservative treatment of this kind of tumour.

\section{CONCLUSION}

This neoplasm is rare, most commonly occurring in the late teens and early 20 s, and usually presenting as a cervical polyp.

In order to argue the best therapeutic approach, a multidisciplinary team, consisting of oncologists, gynaecologists and, sometimes, paediatricians is required to decide the timely use of surgery, radiotherapy and chemotherapy.

More and larger studies and longer follow up are needed to determine the long-term fertility outcomes and survival outcomes in these patients, which is difficult due to low incidence of ERMS.

\section{ACKNOWLEDGEMENTS}

We would like to thank Dr. Eduardo Silva Ferreira, for providing the Microscopic examination figures.

\author{
Funding: No funding sources \\ Conflict of interest: None declared \\ Ethical approval: Not required
}

\section{REFERENCES}

1. Behtash N, Mousavi A, Tehranian A, Khanafshar N, Hanjani P. Embryonal rhabdomyosarcoma of the uterine cervix: case report and review of the literature. Gynecol Oncol. 2003;91:452-5.

2. Daya DA, Scully RE. Sarcoma botryoides of the uterine cervix in young women: a clinicopathological study of 13 cases. Gynecol Oncol. 1988;29:290-304.

3. Ghaemmaghami F, Zarchi MK, Ghasemi M. Lower genital tract rhabdomyosarcoma: case series and literature review. Arch Gynecol Obstet. 2008;278:65-9.

4. Scaravilli G, Simeone S, Orabona GDA, Capuano S, Serao M, Rossi R et al. Case report of a sarcoma botryoides of the uterine cervix in fertile age and literature review. Arch Gynecol Obstet. 2009;280:8636.

5. Dehner L.P., Jarzembowski J.A., Hill D.A. Embryonal rhabdomyosarcoma of the uterine cervix: a report of 14 cases and a discussion of its unusual clinicopathological associations. Mod. Pathol. 2012;25(4):602-14. 
6. Bernal KL, Fahmy L, Remmenga S, Julia B, Baker J. Embryonal rhabdomyosarcoma (sarcoma botryoides) of the cervix presenting as a cervical polyp treated with fertility-sparing surgery and adjuvant chemotherapy. Gynecol Oncol. 2004;95:243-6.

7. Melo A, Amorim-Costa C, Pires MC, Fernandes D, Soares M, Cambão M, et al. Botryoid rhabdomyosarcoma of the uterine cervix: a case report. Journal Obstet and Gynaecol. 2012;7:709-711.
8. Ayas S, Uygur L, Bostanci E, Gürbüz A. A successful pregnancy during the treatment of cervical sarcoma botryoides and advantage of fertility sparing management: A case report: Iran J Reprod Med. 2015;13(2):113-6.

9. Bouchard-Fortier G, Kim RH, Allen L, Gupta A, May T. Fertility-sparing surgery for the management of young women with embryonal rhabdomyosarcoma of the cervix: A case series. Gynecol Oncol Rep. 201626;18:4-7.

Cite this article as: Gomes AR, Leite PB, Rocha JS, Enes PV, Carvalho JI, Pinto AR, et al. Embryonal rhabdomyosarcoma of the uterine cervix: a rare case report. Int J Reprod Contracept Obstet Gynecol 2017;6:309-12. 